\title{
A LARGE DEVIATION PRINCIPLE FOR THE REDUCTION OF PRODUCT REPRESENTATIONS
}

\author{
N. G. DUFFIELD
}

(Communicated by George C. Papanicolaou)

\begin{abstract}
A large deviation principle is proved for a family of measures $\left\{\mathbb{L}_{n}: n=1,2, \ldots\right\}$ derived from the multiplicities occurring in the decomposition into irreducible components of $n$-fold tensor products of representations of arbitrary compact semisimple Lie groups.
\end{abstract}

\section{INTRODUCTION}

In [1], Cegla, Lewis, and Raggio proved a large deviation principle [2, 3] for a family of measures derived from the multiplicities occurring in the decomposition of tensor products of representations of $S U(2)$ into irreducible components. They used their result in the analysis of the thermodynamics of quantum mean-field spin systems. In this paper we extend their result to cover any compact semisimple Lie group. In another paper [4] we use our large deviation principle to obtain a variational expression for thermodynamic limit of the free energy for mean-field systems based upon arbitrary compact semisimple Lie groups. (But see also [5,6] for a different approach to similar problems.)

We now give a brief sketch of the results of [1]. This will be done in a more general setting than that of [1] in order to show how our own result proceeds naturally from it. For an arbitrary compact semisimple Lie group $G$, denote the set of finite dimensional unitary irreducible representations by $\left\{\Phi^{J}: J \in \mathcal{J}\right\}$, where $\mathscr{J}$ is some countable index set. Pick some $j$ in $\mathscr{J}$ and $n$ in $\mathbb{N}$ and form the unitary representation

$$
\Phi_{n}^{j}(g)=\underbrace{\Phi^{j}(g) \otimes \cdots \otimes \Phi^{j}(g)}_{n \text { times }}, \quad g \in G .
$$

Received by the editors November 10, 1988 and, in revised form, August 3, 1989.

1980 Mathematics Subject Classification (1985 Revision). Primary 60F10; Secondary 17B10, $17 \mathrm{~B} 20$

Key words and phrases. Large deviations, Lie groups, reduction of representations.

This research was supported by a European Science Exchange Fellowship of the Royal Society, London. 
For $n>1, \Phi_{n}^{j}$ is reducible and

$$
\Phi_{n}^{j}=\bigoplus_{J \in D_{n}^{j}} \bigoplus_{k=1}^{b^{j}(n, J)} \Phi^{J, k}
$$

where $D_{n}^{j}$ is a finite subset of $\mathscr{J}, \Phi^{J, k}$ is a copy of $\Phi^{J}$, and $b^{j}(n, J)$ is the multiplicity of $\Phi^{J}$ in the decomposition.

In fact, it turns out that $\mathcal{J}$ is a subset of a certain vector space (which we will later call $\left.\left(\mathrm{h}_{\mathbb{R}}\right)^{\prime}\right)$ whose dimension is equal to the rank, $l$, of $G$. $\mathscr{J}$ is the intersection of a certain lattice $\mathscr{I}$ with a certain cone $D$ in $\left(\mathrm{h}_{\mathbb{R}}\right)^{\prime}$.

Define the family of probability measures $\left\{\mathbb{L}_{n}^{j}: n=1,2, \ldots\right\}$ on $D$ by

$$
\mathbb{L}_{n}^{j}[A]=\left(\sum_{J \in D_{n}^{j}} b^{j}(n, J)\right)^{-1} \sum_{J \in D_{n}^{j}: J / n \in A} b^{j}(n, J)
$$

for $A$ a Borel subset of $D$.

When $G=S U(2)$ then $l=1,\left(\mathrm{~h}_{\mathbb{R}}\right)^{\prime}=\mathbb{R}, D=[0, \infty)$ and

$$
\mathscr{J}=\left\{0, \frac{1}{2}, 1, \frac{3}{2}, \ldots\right\} \text {. }
$$

$\Phi^{J}$ is the unitary irreducible representation of $S U(2)$ which acts on $\mathbb{C}^{2 J+1}$ and $D_{n}^{j}=\left\{0,1, \ldots, \frac{n}{2}\right\}$ for $2 n j$ even, while $D_{n}^{j}=\left\{\frac{1}{2}, \frac{3}{2}, \ldots, \frac{n}{2}\right\}$ for $2 n j$ odd.

We state the result of [1] within our general framework.

Theorem 1.1. With the notation above for the case $G=S U(2)$, the family of measures $\left\{\mathbb{L}_{n}^{j}: n=1,2, \ldots\right\}$ satisfies a large deviation principle with constants $\{n\}$ and rate function $I^{j}: D \rightarrow[0, \infty]:$

$$
I^{j}(r)=\sup _{t \in D}\left\{r t-\log \left(\frac{\chi^{j}\left(e^{t S^{z}}\right)}{d^{j}}\right)\right\},
$$

where $\chi^{j}$ is the character of the representation $\Phi^{j}$ and $d^{j}$ is its dimension.

The rate function $J^{j}$ can be viewed as a specific entropy density for the limiting distribution of the multiplicities.

The contribution of the present paper to the theory is to prove an analogous result for arbitrary compact semisimple $G$. We state and prove the main result (Theorem 2.1) in the next section. The first step is to find the cumulant generating function for the sequence of measures (1.3). This is done in Lemma 2.2 and Proposition 2.5(i). In Proposition 2.5(ii) it is shown that the cumulant generating functional is differentiable. By a standard result in the theory of large deviations, Proposition B.1, this allows us to conclude the main result.

In order to make the paper reasonably self-contained, a summary of the representation theory of semisimple Lie groups $[7,8]$ is given, while an outline 
of the theory of large deviations is given in Appendix B. Some technical proofs in $\S 2$ are deferred to Appendix C.

\section{THE LARGE DEVIATION PRINCIPLE}

To avoid a major diversion into the details of representation theory we refer the reader to Appendix A. Let $G$ be a compact semisimple Lie group. Let $l$ denote the rank of $G$, i.e. the dimension of a maximal abelian, or Cartan, subgroup $H$, and let $E$ denote the set $\{1,2, \ldots, l\}$. Denote by $\mathrm{h}$ the Lie algebra of $H$, and let $\left(\mathrm{h}_{\mathbb{R}}\right)^{\prime}$ denote the real dual of $i$ h. Let $D$ denote the dominant elements of $\left(h_{\mathbb{R}}\right)^{\prime}$. For $\lambda$ a dominant weight in $\left(h_{\mathbb{R}}\right)^{\prime}$, let $\Phi^{\lambda}$ be the irreducible representation of $G$ with maximal weight $\lambda$ (as in Proposition A.1). Define the unitary representation $\Phi_{n}^{\lambda}$ of $G$ by

$$
\Phi_{n}^{\lambda}(g)=\underbrace{\Phi^{\lambda}(g) \otimes \cdots \otimes \Phi^{\lambda}(g)}_{n \text { times }}, \quad g \in G .
$$

For $n>1, \Phi_{n}^{\lambda}$ is reducible and decomposes into a direct sum,

$$
\Phi_{n}^{\lambda}=\bigoplus_{\mu \in D_{n}^{\lambda}} \bigoplus_{k=1}^{b^{\lambda}(n, \mu)} \Phi^{\mu, k},
$$

where $D_{n}^{\lambda} \subset D$ is the set of dominant analytically integral forms whose members label the irreducible representations occurring in the decomposition, $b^{\lambda}(n, \mu)$ is the multiplicity of the representation with maximal weight $\mu$ in the decomposition, and $\Phi^{\mu, k}$ is a copy of the irreducible representation $\Phi^{\mu}$. We denote by $\chi^{\lambda}$ and $\chi_{n}^{\lambda}$ the characters of the representations $\Phi^{\lambda}$ and $\Phi_{n}^{\lambda}$ respectively. We define a family of measures $\left\{\mathbb{L}_{n}^{\lambda}: n=1,2, \ldots\right\}$ on $D$ by

$$
\mathbb{L}_{n}^{\lambda}[A]=\frac{1}{B^{\lambda}(n)} \sum_{\mu \in D_{n}^{\lambda}: \mu / n \in A} b^{\lambda}(n, u)
$$

for $A$ a Borel subset of $D$ and

$$
B^{\lambda}(n)=\sum_{\mu \in D_{n}^{\lambda}} b^{\lambda}(n, u)
$$

We now state the main theorem of the paper.

Theorem 2.1. The sequence $\left\{\mathbb{L}_{n}^{\lambda}: n=1,2, \ldots\right\}$ satisfies a large deviation principle on $\left(\mathrm{h}_{\mathbb{R}}\right)^{\prime}$ with constants $\{n\}$ and rate function

$$
I^{\lambda}(\mu)=\sup _{h \in j^{-1} D}\left\{\mu(h)-\tilde{c}^{\lambda}(h)\right\}
$$

where $\tilde{c}^{\lambda}: j^{-1} D \mapsto[0, \infty]$ is given by

$$
\tilde{c}^{\lambda}(h)=\log \frac{\chi^{\lambda}\left(e^{h}\right)}{d^{\lambda}},
$$


where $d^{\lambda}$ is the dimension of the representation $\Phi^{\lambda}$ and the canonical linear map $j: i \mathrm{~h} \rightarrow\left(\mathrm{h}_{\mathbb{R}}\right)^{\prime}$ is as defined in (A.4).

The proof of the theorem is carried out in stages. In Lemma 2.2 we calculate the asymptotics of $B^{\lambda}(n)$. Next we calculate the cumulant generating functional $c^{\lambda}(h)=\lim _{n \rightarrow \infty} c_{n}^{\lambda}(h)$ on $\mathrm{h}_{\mathbb{R}}$, where

$$
c_{n}^{\lambda}(h)=\frac{1}{n} \log \int_{\left(\mathrm{h}_{\mathbf{R}}\right)^{\prime}} \mathbb{L}_{n}(d \rho) e^{\rho(h)} .
$$

In Proposition 2.3 we calculate the restriction $\tilde{c}^{\lambda}$ of $c^{\lambda}$ to $j^{-1} D$. In Proposition 2.5 we extend this result to find $c^{\lambda}$ on whole of $h_{\mathbb{R}}$, and show that it is differentiable. This condition allows us to conclude the statement of the theorem on general grounds (Proposition B.1). For notational clarity, we henceforth omit the superscript $\lambda$ on $c_{n}^{\lambda}, c^{\lambda}$ and $\tilde{c}^{\lambda}$.

Lemma 2.2. The asymptotics of $B^{\lambda}(n)$.

$$
B^{\lambda}(n) \leq\left(d^{\lambda}\right)^{n} \leq a_{2}\left(a_{3}+n\right)^{a_{4}} B^{\lambda}(n)
$$

for some positive constants $a_{1}, a_{3}$ and $a_{4}$.

Proof. Equating the dimensions of both sides of (2.2)

$$
\left(d^{\lambda}\right)^{n}=\sum_{\mu \in D_{n}^{\lambda}} b^{\lambda}(n, \mu) d^{\mu} .
$$

Clearly $d^{\mu} \geq 1$, and so $B^{\lambda}(n) \leq\left(d^{\lambda}\right)^{n}$. To obtain upper bounds for the $d^{\mu}$, we note that by Proposition A.3 the highest maximal weight for representations in the decomposition (2.2) is $n \lambda$. Thus $n \lambda-\mu \geq 0$ for all $\mu$ in $D_{n}^{\lambda}$. Since $n \lambda$ and $\mu$ are both dominant we have that $\langle n \lambda, n \lambda-\mu\rangle \geq 0$ and $\langle\mu, n \lambda-\mu\rangle \geq 0$ for all $\mu$ in $D_{n}^{\lambda}$. Adding these, $\langle n \lambda, n \lambda\rangle \geq\langle\mu, \mu\rangle$. From the Weyl dimension formula (Proposition A.2), with $\delta$ the magic weight defined in (A.13),

$$
d^{\mu}=\prod_{\alpha \in \Delta^{+}}\left(1+\frac{\langle\mu, \alpha\rangle}{\langle\delta, \alpha\rangle}\right) \leq \prod_{\alpha \in \Delta^{+}}\left(1+a_{1}\langle\mu, \mu\rangle^{\frac{1}{2}}\langle\alpha, \alpha\rangle^{\frac{1}{2}}\right) \leq\left(a_{2}+a_{3} n\right)^{a_{4}}
$$

for some positive constants $a_{1}, \ldots, a_{4}$, the first inequality following from the Cauchy-Schwarz inequality. Thus (2.8) follows immediately.

Proposition 2.3. The restricted cumulant generating functional. Let $h \in j^{-1} D$. Then

$$
\tilde{c}(h)=\lim _{n \rightarrow \infty} c_{n}(h)=\log \left(\frac{\chi^{\lambda}\left(e^{h}\right)}{d^{\lambda}}\right),
$$

convergence being uniform over $j^{-1} D$.

Proof. Let $h$ be as above.

$$
\left(\chi^{\lambda}\left(e^{h}\right)\right)^{n}=\chi_{n}^{\lambda}\left(e^{h}\right)=\sum_{\mu \in D_{n}^{\lambda}} b^{\lambda}(n, \mu) \chi^{\mu}\left(e^{h}\right) .
$$


Let $Q^{\mu}$ denote the set of weights of the representation $\Pi^{\mu}$. Then

$$
e^{\mu(h)} \leq \chi^{\mu}\left(e^{h}\right)=\sum_{\sigma \in Q^{\mu}} m^{\mu}(\sigma) e^{\sigma(h)} \leq d^{\mu} e^{\mu(h)} .
$$

The equality is merely (A.11). Since $h \in \mathrm{h}_{\mathbb{R}}, \sigma(h)$ is real, and the first inequality follows from Proposition A.1(ii). $j h$ is dominant, so by Proposition A.1(iii) $\mu(h) \geq \sigma(h)$ for $\sigma \in Q^{\mu}$; and since $\sum_{\sigma \in Q^{\mu}} m^{\mu}(\sigma)=d^{\mu}$, the second inequality follows. Thus

$$
\sum_{\mu \in D_{n}^{\lambda}} b^{\lambda}(n, \mu) e^{\mu(h)} \leq\left(\chi^{\lambda}\left(e^{h}\right)\right)^{n} \leq \sum_{\mu \in D_{n}^{\lambda}} b^{\lambda}(n, \mu) d^{\mu} e^{\mu(h)} .
$$

Using (2.10) the upper bound in (2.14) can be rewritten

$$
\left(\chi^{\lambda}\left(e^{h}\right)\right)^{n} \leq \sum_{\mu \in D_{n}^{\lambda}} b^{\lambda}(n, \mu) a_{2}\left(a_{3}+n\right)^{a_{4}} e^{\mu(h)} .
$$

Thus combining (2.8), (2.14), and (2.15) with (2.3), the definition of $\mathbb{L}_{n}^{\lambda}$,

$$
\left|\frac{1}{n} \log \int_{\left(\mathrm{h}_{\mathbf{R}}\right)^{\prime}} \mathbb{L}_{n}(d \rho) e^{n \rho(h)}-\log \left(\frac{\chi^{\lambda}\left(e^{h}\right)}{d^{\lambda}}\right)\right|=O\left(n^{-1} \log n\right)
$$

Therefore, passing to the limit as $n \rightarrow \infty$ we obtain (2.11), the limit existing uniformly in $j^{-1} D$.

The following lemma gives us a decomposition of $\left(h_{\mathbb{R}}\right)^{\prime}$ which will enable us to extend the result of the previous proposition to the whole of $\left(h_{\mathbb{R}}\right)^{\prime}$. First, for $I, J \subseteq E$ with $I \cap J=\varnothing$, we define

$$
D(I, J)=\left\{x \in\left(\mathrm{h}_{\mathbb{R}}\right)^{\prime}: x=\sum_{i \in I} l_{i} \lambda_{i}+\sum_{j \in J} a_{i} \alpha_{i} ; \quad l_{i}>0, a_{i}<0\right\} .
$$

Lemma 2.4. Every $x$ in $\left(\mathrm{h}_{\mathbb{R}}\right)^{\prime}$ is an element of $D(I, J)$ for some $I$ and $J$ depending on $x$.

Proof. For each $x$ there is a point $M(x)$ in $D$ such that

$$
\inf _{y \in D}\|x-y\|=\|M(x)-x\| .
$$

The infimum is clearly attained, since $\inf _{y \in D}\|x-y\|=\inf _{y \in D_{K}}\|x-y\|$, where $D_{K}$ is the compact set $\{y \in D:\|x-y\| \leq\|x\|+K\} . D$ is a cone and hence convex, so $M(x)$ is the unique such point; and since $M(x) \in D$ we can write $M(x)=\sum_{i \in I(x)} l_{i} \lambda_{i}$ with $l_{i}>0$ for some subset $I(x)$ of $E$. We write $x-$ $M(x)=\sum_{i \in E} a_{i} \alpha_{i}$ for some coefficients $a_{i}$, and for $t \geq 0$ let $M(x, t)=$ $M(x)+t \sum_{i \in E} \varepsilon_{i} \lambda_{i}$ represent an arbitrary perturbation of $M(x)$ within $D$, i.e.

$$
\varepsilon_{i}>0: i \in E \backslash I(x) \text { with } \varepsilon_{i} \in \mathbb{R} \text { otherwise. }
$$

Then with $\|x\|=\langle x, x\rangle^{\frac{1}{2}}$ for the inner product $\langle\cdot, \cdot\rangle$ defined in (A.4)

$$
\|x-M(x, t)\|=\left\|\sum_{E}\left(a_{i} \alpha_{i}-t \varepsilon_{i} \lambda_{i}\right)\right\|,
$$


so that by $(\mathrm{A} .8)$

$$
\lim _{t \backslash 0} \frac{d}{d t}\|x-M(x, t)\|^{2}=-\sum_{i \in E} a_{i} \varepsilon_{i}\left\langle\alpha_{i}, \alpha_{i}\right\rangle .
$$

But since $M(x)$ is the closest point in $D$ to $x,(2.21)$ must be nonnegative. Hence $a_{i}=0$ for $i \in I(x)$, while $a_{i}<0$ for $i \in E \backslash I(x)$. Adding the expressions for $M(x)$ and $x-M(x)$, we get the stated result.

The point of the lemma is shown by the following proposition, whose proof, being elementary but tedious, is given in Appendix C.

\section{Proposition 2.5.}

(i) The cumulant generating functional $c$ is calculated from its restriction to $j^{-1} D$ as follows:

$$
c(h)=\tilde{c}\left(j^{-1} M(j h)\right) ;
$$

(ii) $c$ is differentiable throughout $\left(\mathrm{h}_{\mathbb{R}}\right)^{\prime}$.

Proof of Theorem 2.1. Combine Propositions 2.5 and B.1 to conclude immediately that $\left\{\mathbb{L}_{n}: n=1,2, \ldots\right\}$ satisfies a large deviation principle with constants $\{n\}$ and rate function $I^{\lambda}:\left(\mathrm{h}_{\mathbb{R}}\right)^{\prime} \rightarrow[0, \infty]$,

$$
I^{\lambda}(\mu)=\sup _{h \in \mathrm{h}_{\mathbf{R}}}\{\mu(h)-c(h)\} .
$$

Lemma C. 1 tells us that the derivatives of $c \circ j^{-1}$ normal to the boundaries of $D$ are zero.

$$
\nabla_{\alpha_{i}} c \circ j^{-1} \mid \bigcup_{J \subseteq E \backslash\{i\}} D(J, 0)=0 .
$$

But writing any $\alpha$ as $\sum_{E} a_{i} \alpha_{i}$, one sees from (2.7) that $c_{n}\left(j^{-1} \alpha\right)$, and hence $c\left(j^{-1} \alpha\right)$, are nondecreasing functions of each of the $a_{i}$ 's. Thus the range of $\nabla c \circ j^{-1}$ is attained on $D$, and so the supremum in (2.23) can be restricted to $j^{-1} D$.

Remark 2.6. By Proposition A.1(iii) we see that the essential domain of $I^{\lambda}$ is

$$
\text { ess dom } I^{\lambda}=D \cap\left\{x \in\left(\mathrm{h}_{\mathbb{R}}\right)^{\prime}: x=\lambda-\sum_{E} r_{i} \alpha_{i}: r_{i} \geq 0\right\} \text {. }
$$

Elsewhere $I^{\lambda}$ takes the value $+\infty$.

\section{Discussion}

We briefly contrast the work in this paper with a subsidiary result at the end of $\S 2$ in [1]. In the latter case a purely algebraic decomposition of tensor products of arbitrary finite dimensional $C-^{*}$ algebra was obtained in terms of polynomial in representatives of the Lie algebra $S U(2)$. This is not a group decomposition into irreducible representations in the standard sense, and is 
as such quite distinct from the present paper: Theorem 2.1. is based on the standard decomposition of tensor products into irreducible representations.

\section{APPENDIX A. REPRESENTATION THEORY}

Representations and weights. In this section we provide for reference a summary of the necessary facts of representation theory $[7,8]$. Let $G$ be a compact semisimple Lie group with Lie algebra g. $G \subseteq U(n)$ for some $n$, and so setting $\mathbf{g}_{\mathbb{R}}=i \mathbf{g}$ we define the complexification $\mathbf{g}^{\mathbb{C}}=\mathbf{g}_{\mathbb{R}} \oplus i \mathbf{g}_{\mathbb{R}}$ in terms of matrices. Let $H$ be a Cartan subgroup of $G$, i.e. a maximal abelian subgroup, with corresponding subalgebra $h$. Similarly, we define $h_{\mathbb{R}}$ and $h^{\mathbb{C}}$.

Let $\Phi$ be a representation of $G$ on a finite dimensional complex vector space $V$, and let $\phi$ be its differential acting on $\mathbf{g}$. We use the same symbol $\phi$ to denote the extension of $\phi$ to $\mathbf{g}^{\mathbb{C}}$. Since $\mathrm{h}$ is abelian, the $\left\{\phi(h): h \in \mathrm{h}^{\mathbb{C}}\right\}$ commute. Hence there exists a simultaneous eigenspace decomposition of $V$ for all of $\phi\left(h^{\mathbb{C}}\right)$, with the eigenvalues given by linear functionals on $h^{\mathbb{C}}$ which are real on $h_{\mathbb{R}}$ :

$$
V=\sum_{\lambda \in \Lambda} V_{\lambda},
$$

where $\Lambda$ is a finite subset of $\left(h_{\mathbb{R}}\right)^{\prime}$, the dual of $h^{\mathbb{C}}$, and

$$
V_{\lambda}=\left\{v \in V: \phi(h) v=\lambda(h) v \quad \forall h \in \mathrm{h}^{\mathbb{C}}\right\} .
$$

The $\lambda$ are called weights and the $V_{\lambda}$ are the associated weight spaces. The weights of the adjoint representation are called roots.

Root spaces. Let ad denote the adjoint representation of $G$ acting on $\mathbf{g}$. The complex bilinear form

$$
K\left(h_{1}, h_{2}\right)=\operatorname{trace}\left(\operatorname{ad} h_{1} \operatorname{ad} h_{2}\right)
$$

on $h^{\mathbb{C}}$ is positive definite on $h_{\mathbb{R}}$ and thus defines an inner product there. Thus by duality we can define the bijection $j: \mathrm{h}_{\mathbb{R}} \mapsto\left(\mathrm{h}_{\mathbb{R}}\right)^{\prime}$

$$
\left(j h_{1}\right)\left(h_{2}\right)=K\left(h_{1}, h_{2}\right)
$$

and an inner product $\langle\cdot, \cdot\rangle$ on $\left(h_{\mathbb{R}}\right)^{\prime}$ :

$$
\langle x, y\rangle=K\left(j^{-1} x, j^{-1} y\right) .
$$

We topologize $h_{\mathbb{R}}$ and $\left(h_{\mathbb{R}}\right)^{\prime}$ with the norms associated with their respective scalar products. We denote these by $\|\cdot\|$. Define an ordering among the roots as follows: fix $v_{0} \in \mathrm{h}$ such that $\alpha\left(v_{0}\right) \neq 0$ for all nonzero $\alpha$ in $\Delta$. For $x, y \in\left(\mathrm{h}_{\mathbb{R}}\right)^{\prime}$ we say that $x \geq y$ if $x\left(v_{0}\right) \geq y\left(v_{0}\right)$. Define $\Pi$, the set of simple roots, to be those positive roots (with respect to the ordering above) which cannot be decomposed as a sum of positive roots. $\Pi$ has $l$ elements, which we denote $\alpha_{i}: i \in E$. The $\alpha_{i}$ span $\left(\mathrm{h}_{\mathbb{R}}\right)^{\prime}$ and furthermore
(A.6)
$\left\langle\alpha_{i}, \alpha_{j}\right\rangle \leq 0 \quad i \neq j$. 
The Weyl group. Define the reflections $\left\{w_{i}: i \in E\right\}$ in $\left(\mathrm{h}_{\mathbb{R}}\right)^{\prime}$ by

$$
w_{i}(\beta)=\beta-2 \frac{\left\langle\beta, \alpha_{i}\right\rangle}{\left\langle\alpha_{i}, \alpha_{i}\right\rangle} \alpha_{i} .
$$

The Weyl group $W$ is defined to be that generated by these reflections. Under the action of $W,\left(h_{\mathbb{R}}\right)^{\prime}$ divides up into simplical cones (the Weyl chambers) whose boundaries are invariant hyperplanes of the reflections in $W$.

Dominant and integral forms. Define $D$, the set of dominant forms, by $D=$ $\left\{x \in\left(\mathrm{h}_{\mathbb{R}}\right)^{\prime}:\left\langle x, \alpha_{i}\right\rangle \geq 0 \forall \alpha_{i} \in \Pi\right\} . D$ comprises the dominant Weyl chamber: the convex region bounded by the invariant hyperplanes of $\left\{w_{i}: i=1, \ldots, l\right\}$. Define a basis $\left\{\lambda_{i}: i=1, \ldots, l\right\}$ in $\left(\mathrm{h}_{\mathbb{R}}\right)^{\prime}$ by

$$
2 \frac{\left\langle\lambda_{i}, \alpha_{j}\right\rangle}{\left\langle\alpha_{j}, \alpha_{j}\right\rangle}=\delta_{i j} .
$$

Define the set of analytic integral forms $\mathscr{I}$ to be those forms $\lambda$ for which there exists a character $\zeta_{\lambda}$ of $H$ such that

$$
\zeta_{\lambda}\left(e^{h}\right)=e^{\lambda(h)}
$$

for all $h$ in $h . \mathscr{I}$ itself is a sublattice of the lattice in $\left(h_{\mathbb{R}}\right)^{\prime}$ generated by $\left\{\lambda_{i}: i=1, \ldots, l\right\}$. The weights occurring in the decomposition (A.1) are in $\mathscr{I}$. Thus we write the character of the representation $\Phi$ on $H$

$$
\chi\left(e^{h}\right)=\sum_{\lambda \in \Lambda} m(\lambda) \zeta_{\lambda}\left(e^{h}\right),
$$

where the multiplicity $m(\lambda)$ is the dimension of the space $V_{\lambda}$. We note at this point that $m(\mu)=m(w \mu)$ for $w \in W$. Since $\chi(\cdot)=\operatorname{trace} \Phi(\cdot)$, then by $(\mathrm{A} .1)$ and (A.2) we can analytically extend the character to $H^{\mathbb{C}}$, the group obtained by exponentiation of $h^{\mathbb{C}}$, and write

$$
\chi\left(e^{h}\right)=\sum_{\lambda \in \Lambda} m(\lambda) e^{\lambda(h)} .
$$

We now state the three main theorems which we shall use to prove our result.

Proposition A.1. Given a compact semisimple Lie group $G$, then

(i) the associated dominant analytic integral forms are in one-one correspondence with the irreducible representations of $G$ (up to unitary equivalence). The correspondence is that the form $\lambda \in \mathscr{I} \cap D$ is the maximal weight (with respect to given ordering $\geq$ ) occurring in the representation: we label the latter $\Phi^{\lambda}$.

(ii) The weight space $V_{\lambda}$ of the maximal weight is one-dimensional.

(iii) Every other weight occurring in the representation is of the form $\lambda$ $\sum_{i=1}^{l} n_{i} \alpha_{i}$ with $n_{i}$ a nonnegative integer and $\alpha_{i} \in \Pi$. 
Proposition A.2. The Weyl dimension formula. The dimension $d^{\lambda}$ of the space $V$ in which the representation $\Phi_{\lambda}$ acts is

$$
d^{\lambda}=\frac{\Pi_{\alpha \in \Delta^{+}}\langle\lambda+\delta, \alpha\rangle}{\Pi_{\alpha \in \Delta^{+}}\langle\delta, \alpha\rangle},
$$

where $\delta$ is the magic weight

$$
\text { (A.13) } \delta=\sum_{E} \lambda_{i}
$$

and $\Delta^{+}$is the finite set of positive roots: $\{\alpha \in \Delta: \alpha \geq 0\}$.

Proposition A.3. In the decomposition into irreducible representations of the tensor product $\Phi^{\lambda^{1}} \otimes \Phi^{\lambda^{2}}$, the highest maximal weight occurring is for the representation $\Phi^{\lambda^{1}+\lambda^{2}}$.

\section{APPENDIX B. THE THEORY OF LARGE DEVIATIONS}

Definition. Let $\left\{\mathbb{K}_{n}: n=1,2, \ldots\right\}$ be a sequence of probability measures on the Borel subsets of a complete separable metric space $E$ and $\left\{V_{n}\right\}$ a divergent sequence of positive numbers. We say that $\left\{\mathbb{K}_{n}\right\}$ satisfies a large deviation principle with constants $\left\{V_{n}\right\}$ and rate function $I: E \rightarrow[0, \infty]$ if the following conditions hold:

(LD1) $I$ is lower semicontinuous.

(LD2) For each $m<\infty,\{x: I(x) \leq m\}$ is compact.

(LD3) For each closed subset $C$ of $E$

$$
\limsup _{n \rightarrow \infty} \frac{1}{V_{n}} \log \mathbb{K}_{n}(C) \leq-\inf _{x \in C} I(x) .
$$

(LD4) For each open subset $G$ of $E$

$$
\liminf _{n \rightarrow \infty} \frac{1}{V_{n}} \log \mathbb{K}_{n}(G) \geq-\inf _{x \in G} I(x) .
$$

Proposition B.1 [3, Theorem II.6.1]. Adopting the notation above, let $E$ be a finite dimensional vector space with dual $E^{\prime}$. Define $c_{n}: E^{\prime} \rightarrow \mathbb{R}$ by

$$
c_{n}(y)=\frac{1}{V_{n}} \log \int_{E} \mathbb{L}_{n}(d x) e^{V_{n} y(x)} .
$$

Assume that

(i) $c_{n}$ is finite for all $n$.

(ii) $c(y)=\lim _{n \rightarrow \infty} c_{n}(y)$ exists and is finite for all $y$ in $E$.

(iii) $c(y)$ is differentiable for all $y$ in $E$.

Then the sequence $\left\{\mathbb{L}_{n}: n=1,2, \ldots\right\}$ satisfies a large deviation principle with constants $\left\{V_{n}\right\}$ and rate function

$$
I(x)=\sup _{y \in E^{\prime}}\{y(x)-c(y)\} .
$$




\section{APPENDIX C. EXTENSION AND DIFFERENTIABILITY OF $c$}

Proof of Proposition 2.5(i). Let $h \in\left(h_{\mathbb{R}}\right)^{\prime}$ and set $x=j h$. By Lemma 2.4, $x \in D(I(x), J(x))$ for some $I(x), J(x) \subseteq E$. More precisely, let $x=y+z$, with $y=M(x) \in D$. Since $z=\sum_{E \backslash I(x)} z_{i} \alpha_{i}$ with $z_{i} \leq 0$, then for $\lambda \in D$, $\langle\lambda, z\rangle=\lambda\left(j^{-1} z\right) \leq 0$. Hence by inspection of $(2.7), c_{n}(h) \leq \tilde{c}_{n}\left(j^{-1} M(j h)\right)$, so that

$$
\limsup _{n \rightarrow \infty} c_{n}(h) \leq \tilde{c}\left(j^{-1} M(j h)\right)
$$

If we can prove that

$$
\liminf _{n \rightarrow \infty} c_{n}(h) \geq \tilde{c}\left(j^{-1} M(j h)\right)
$$

then we are done.

Considering the relations (A.8), then clearly one can pick some dominant $q$ such that $q+z$ is dominant. For $\tau \geq 0$ and $t$ real

$$
x_{\tau}(t)=y-t z+\frac{\tau(t+1)}{\tau+1} q .
$$

Then $x_{\tau}(-1)=x$, and for $t$ in $[0, \tau], x_{\tau}(t)$ is dominant. Suppose (C.2) is not satisfied. Then there exists $\varepsilon^{\prime}>0$ and a subsequence of integers, which we also denote by $\{n\}$, such that $c_{n}(h) \leq \tilde{c}\left(j^{-1} y\right)-\varepsilon^{\prime}$ for $n$ sufficiently large. But since $\lim _{\tau \rightarrow 0} x_{\tau}(0)=y$ and $c_{n}$ converges to $\tilde{c}$ uniformly on $j^{-1} D$, then there exists $\varepsilon>0$ such that

$$
c_{n}(x) \leq c_{n}\left(x_{\tau}(0)\right)-\varepsilon
$$

for $n$ sufficiently large.

Now $j$ is linear, $x_{\tau}(\cdot)$ is affine and $c_{n}(\cdot)$ is convex. Thus

$$
c_{n}\left(j^{-1} x_{\tau}(0)\right) \leq \frac{t}{1+t} c_{n}\left(j^{-1} x_{\tau}(-1)\right)+\frac{1}{1+t} c_{n}\left(j^{-1} x_{\tau}(t)\right)
$$

and

$$
c_{n}\left(j^{-1} x_{\tau}(0)\right) \geq c_{n}\left(j^{-1} x_{\tau}(t)\right)-t \frac{d}{d t} c_{n}\left(j^{-1} x_{\tau}(t)\right) .
$$

Combining (C.4), (C.5), and (C.6) we see that

$$
\frac{d}{d t} c_{n}\left(j^{-1} x_{\tau}(t)\right) \geq \varepsilon
$$

for all $n$ sufficiently large, and since $\tilde{c}$ is, by examination, differentiable on $j^{-1} D$, we can use Griffiths's lemma (e.g. [3]) to conclude that

$$
\left.\lim _{\tau \rightarrow 0} \frac{d}{d t} \tilde{c}\left(j^{-1} x_{\tau}(t)\right)\right|_{t=0} \geq \varepsilon .
$$

By the following lemma, (C.8) is not true, and so (C.2) must hold. Thus $c(h)=$ $\lim _{n \rightarrow \infty} c_{n}(h)$ exists for all $h \in\left(\mathrm{h}_{\mathbb{R}}\right)^{\prime}$ and is given by (2.22).

The following lemma tells us that at a boundary of $D$ the normal derivatives of $c \circ j^{-1}$ vanish. 
Lemma C.1. For all $\tau \geq 0,0 \leq t \leq \tau$, let $x_{\tau}(t)=y-t z+\frac{\tau(t+1)}{\tau+1} q$ be in $D$, with $y \in D(I, \varnothing)$ and $z \in D(\varnothing, J)$, for some $I \subseteq E, J \subseteq E \backslash I$, and some $q \in\left(\mathrm{h}_{\mathbb{R}}\right)^{\prime}$. Then

$$
\left.\lim _{\tau \rightarrow 0} \frac{d}{d t} \tilde{c}\left(j^{-1} x_{\tau}(t)\right)\right|_{t=0}=0
$$

Proof. By (A.10)

$$
\frac{d}{d t} \tilde{c}\left(j^{-1} x_{\tau}(t)\right)=\sum_{\mu \in Q^{\lambda}} m^{\lambda}(\mu) e^{\left\langle\mu, x_{\tau}(t)\right\rangle}\left\langle\mu, \frac{\tau}{\tau+1} q-w\right\rangle,
$$

and so the L.H.S. of (C.9) is just

$$
-\sum_{\mu \in Q^{\lambda}} m^{\lambda}(\mu) e^{\langle\mu, y\rangle}\langle\mu, z\rangle .
$$

Using the symmetry $m^{\lambda}(\mu)=m^{\lambda}(w \mu)$ for $w \in W$, this can be written as

$$
\frac{-1}{\# W} \sum_{w \in W} \sum_{\mu \in Q^{\lambda}} m^{\lambda}(\mu) e^{\langle\mu, w y\rangle}\langle\mu, w z\rangle,
$$

where $\# W$ is the number of elements of $W$.

Let $W_{e}$ (resp. $W_{0}$ ) denote the even (resp. odd) elements of $W$. Then for any odd $w$ in $W, W_{0} w \subset W_{e}$, and $W_{e} w \subset W_{0}$. Thus $\# W_{e}=\# W_{0}$, and $W=W_{e} \cup W_{e} w$. We set $z=\sum_{E \backslash I} z_{i} \alpha_{i}$ and note that by (A.7)

$$
w_{i} \alpha_{i}=-\alpha_{i} \text { and } w_{i} \lambda_{j}=\lambda_{j}: i \neq j \text {. }
$$

Then we can write (C.12) as

(C.14) $\quad \frac{-1}{\# W} \sum_{i \in E \backslash I} z_{i} \sum_{w \in W_{e}} \sum_{\mu \in Q^{\lambda}} m^{\lambda}(\mu)\left(\left\langle\mu, w w_{i} \alpha_{i}\right\rangle e^{\left\langle\mu, w w_{i} y\right\rangle}-\left\langle\mu, w \alpha_{i}\right\rangle e^{\langle\mu, w y\rangle}\right)$

which by virtue of (C.13) is zero.

Proof of Proposition 2.5(ii). Since $c$, as the limit of a sequence of convex functions $c_{n}$, is convex, then within the interior of the regions $j^{-1} D(I, E \backslash I)$ we need only differentiate with respect to the coordinates of the expansion (2.17). At the boundaries between the different regions things are more difficult: a different parametrization is used in each region.

Let $h \in\left(\mathrm{h}_{\mathbb{R}}\right)^{\prime}$ and $u=j h \in D(I(u), J)$. Only the coordinates of $\left\{\alpha_{i}: i \in\right.$ $E \backslash(I \cup J)\}$ are nonzero, so we need only verify the differentiability of the functions

$$
f_{i}(t)=c\left(u_{i}(t)\right) \quad \text { where } u_{i}(t)=u+t \alpha_{i}
$$

for these coordinates. Thus we must show that

$$
\lim _{t \succ 0} f_{i}^{\prime}(t)=\lim _{t \searrow 0} f_{i}^{\prime}(t) \text {. }
$$


When $t<0, M\left(u_{i}(t)\right)=M(u)$ and so $f_{i}^{\prime}(t)=0$. When $t>0$ we must work harder. Write

$$
u_{i}(t)=u+t v+t\left(\alpha_{i}-v\right)
$$

with

$$
v=\sum_{j, k \in E \backslash(I \cup\{i\})}\left\langle\alpha_{i}, \alpha_{j}\right\rangle B_{j k}^{-1} \alpha_{k}
$$

and where $\left\{B_{j k}: j, k \in E \backslash(I \cup\{i\})\right\}$ is the matrix with entries $B_{j k}=\left\langle\alpha_{j}, \alpha_{k}\right\rangle$. We note at this point that by $[7$, p. 90 , Proposition $A]$, the entries of $B^{-1}$ are nonnegative. So by (2.17) and (A.6)

$$
v \in D(\varnothing, E \backslash(I \cup\{i\}))
$$

If we expand $\alpha_{i}-v$ in the basis $\left\{\lambda_{i}: i \in E\right\}$ :

$$
\alpha_{i}-a=\sum_{i \in E} 2 \frac{\left\langle\alpha_{i}, a\right\rangle}{\left\langle\alpha_{i}, \alpha_{i}\right\rangle} \lambda_{i}
$$

then for $l \in E \backslash(I \cup\{i\})$ the coefficient

$$
\begin{aligned}
\left\langle\alpha_{i}-v, \alpha_{l}\right\rangle & =\left\langle\alpha_{i}, \alpha_{l}\right\rangle-\sum_{j, k \in E \backslash(I \cup\{i\})}\left\langle\alpha_{i}, \alpha_{j}\right\rangle B_{j k}^{-1}\left\langle\alpha_{k}, \alpha_{l}\right\rangle \\
& =0
\end{aligned}
$$

while

$$
\left\langle\alpha_{i}-v, \alpha_{i}\right\rangle=\left\langle\alpha_{i}, \alpha_{i}\right\rangle-\sum_{j, k \in E \backslash(I \cup\{i\})}\left\langle\alpha_{i}, \alpha_{j}\right\rangle B_{j k}^{-1}\left\langle\alpha_{k}, \alpha_{i}\right\rangle
$$

The inequality follows from the fact that by the definition of $B, \sum_{i j}\left|\alpha_{i}\right\rangle B_{i j}^{-1}\left\langle\alpha_{j}\right|$ is an orthogonal projection on $\left(\mathrm{h}_{\mathbb{R}}\right)^{\prime}$. (C21) means that $v+t\left(\alpha_{i}-v\right)=$ $\sum_{I \cup\{i\}} l_{i}^{\prime} \lambda_{i}$ for some $l_{i}^{\prime}>0$ and is hence in $D(I \cup\{i\}, \varnothing)$. Therefore

$$
M\left(u_{i}(t)\right)=M\left(M(u)+t\left(\alpha_{i}-v\right)\right)
$$

for $t$ small enough. Furthermore, $M(u)+t\left(\alpha_{i}-v\right)$ is dominant, so applying Lemma C. 1 with $x=u, y=M(u), z=v-\alpha_{i}$, and $q=0$ we obtain that

$$
\lim _{t \searrow 0} f_{i}^{\prime}(t)=0 \text {. }
$$

\section{ACKNOWLEDGMENTS}

Thanks are due to G. A. Raggio for a useful discussion, and to University College, Dublin, where this work was started, for a research fellowship. 


\section{REFERENCES}

1. W. Cegla, J. T. Lewis, and G. A. Raggio, Comm. Math. Phys. 118 (1988), 337-354.

2. S. R. S. Varadhan, Comm. Pure Appl. Math. 19 (1966), 261-286.

3. R. S. Ellis, Entropy, large deviations, and statistical mechanics, Springer, 1985.

4. Classical and thermodynamic limits of generalised quantum spin systems, SFB-123 Heidelberg preprint, no. 505, January 1989; Comm. Math. Phys. (to appear).

5. D. Petz, G. A. Raggio, and A. Verbeure, Comm. Math. Phys. 121 (1989), 271-282.

6. G. A. Raggio and R. F. Werner, Helv. Phys. Acta 62 (1989), 980-1003.

7. H. Samelson, Notes on Lie algebras, Van Nostrand, New York, 1969.

8. A. Knapp, Representation theory of semisimple groups, Princeton Univ. Press, Princeton, NJ, 1986.

Universität Heidelberg, Sonderforschungsbereich 123, Im Neuenheimer Feld 294, D-6900 Heidelberg, Federal Republic of Germany

Current address: Dublin Institute for Advanced Studies, School of Theoretical Physics, 10 Burlington Road, Dublin 4, Ireland 